\title{
The MIMO Surveillance Radars System with High Accuracy Finding 2D Coordinates
}

\author{
Hennadii Khudov ${ }^{1}$, Vitaliy Lishchenko ${ }^{2}$, Henadii Hyshko ${ }^{3}$, Yurii Polonskyi ${ }^{4}$, Irina Khizhnyak ${ }^{5}$, \\ Boris Riabukha ${ }^{6}$ \\ ${ }^{1}$ Department of Radar Troops Tactic, Ivan Kozhedub Kharkiv National Air Force University, Kharkiv, Ukraine, \\ 2345kh_hg@ukr.net \\ ${ }^{2}$ Department of Radar Troops Tactic, Ivan Kozhedub Kharkiv National Air Force University, Kharkiv, Ukraine, \\ lvnpo4ta@gmail.com \\ ${ }^{3}$ Department of Tactic and Military Disciplines, Ivan Kozhedub Kharkiv National Air Force University, Kharkiv, \\ Ukraine, 2345kh_hg@ukr.net \\ ${ }^{4}$ Department of Physics and Radioelectronics, Ivan Kozhedub Kharkiv National Air Force University, \\ Kharkiv, Ukraine, upol_71@gmail.com \\ ${ }^{5}$ Department of Mathematical and Software Automated Control Systems, Ivan Kozhedub Kharkiv National Air \\ Force University, Kharkiv, Ukraine, khizh_ia@ukr.net \\ ${ }^{6}$ Department of Anti-aircraft Missiles Tactic, Ivan Kozhedub Kharkiv National Air Force University, Kharkiv, \\ Ukraine, 2345kh_hg@ukr.net
}

\begin{abstract}
The paper analyses the disadvantages of an existing monostatic radar and the advantages of MIMO (Multiple Input Multiple Output) radar systems for detecting a low radar cross-section of the small-sized aircraft. It was proposed to use several existing unidirectional surveillance radars as elements of a small base synchronous MIMO-radar system in order to improve the quality of detection the aircrafts. This will increase the probability of detecting the small-sized aircraft by exposing them to different angles of spatially spaced radars and using the property of the dependence of aircrafts radar cross-section on the direction of irradiation. In addition the MIMO-radar it is possible to improve the accuracy of the finding 2D coordinates while to surveillance the airspace. The calculation of the quality parameters of detection the aircrafts is carried out by the mathematical modelling method.
\end{abstract}

Key words: MIMO, radar; surveillance; low radar cross section; high accuracy, 2D coordinates, antenna pattern; radar information.

\section{INTRODUCTION}

Every year, the intensity of aviation flights increases, and radar visibility of planes decreases. The safety of flights requires an increase in the quality of radar information [1]-[3]. The task of detecting and radar tracking airborne objects are performed by radar of different generations. The existing radars equipment park includes two-coordinate radars using mechanical rotation to scan the airspace in the azimuth plane [4]-[6].

Such radars have high operational performance and reliability. The number of such radars has a large share compared to all radars. But radar information received by them does not accord the requirements for the quality of information. This is due to the fact that manufacturers constantly improve the shape of airplanes and use modern technologies to reduce radar visibility [4], [7]-[8].

According to an analysis of network-centred wars, local military conflicts and operations in the eastern part of Ukraine, the main trends in the development of new means of air attack are the growing intensity of the use of small-sized aircrafts, usually unmanned aerial vehicles (UAVs) [1]-[2], [9]-[10].

In such circumstances, the improvement of the quality of radar information will be possible by combining separate radar into the multiple-input multiple-output (MIMO)-radars system [3], [5], [9] [11]-[12].

\section{PROBLEM ANALYSIS}

Many different types of multi-site radar systems have been developed in the world. For example, radar systems or individual radars, remote in the space in which the combined processing of radar information is carried out. The development of modern information technology and the using of special high-speed processors and communication lines in signal processing, as well as the using of a new element base, extends the functionality of multi-site radar as a means of radar surveillance. This makes it possible, in real time, to process large amounts of information and increase the speed of information exchange [1], [3], [10]-[12].

The MIMO radar is a special kind of multi-position radar system. The MIMO radar system uses more than one radar source and is able to receive processes and display integral data from all involved radar sources [3], [5], [9] [11]-[12]. 
It is known [11]-[12], that in zones where a large number and intensity of aircraft is possible, they create a rather large group of radar troops. In such hazardous areas, radar surveillance facilities have a dense territorial location and high mobility. In addition, mobile units began to equip two radars. In such circumstances, it would be expedient and economically feasible to use existing radars as elements of the radar system MIMO. In addition, the proximity of the location of radar positions to the separation line can take into account not only the detection of the UAV, but also the determination of their coordinates, including height with the required accuracy [11]-[12].

The MIMO radar system with the potential resultant accuracy of the radar information that can be obtained is higher than that of uncombed radars. the use of systemic effects, including for the low-level MIMO-radar system, created by combining two-coordinate radar observations. The MIMO system includes three stand-alone radars [11]-[12].

The MIMO-radar system with autonomous (cooperative) gain has obvious energy benefits. Positions for receiving such a system can receive echoes from the airspace, which are irradiated by both their own transmitters and transmitters from other positions. One of the key issues determining the possibility of creating such systems is to ensure simultaneous observation of a certain area of space by all spatially-positioned radars. The possibility of implementing coherent air surveillance with the help of spatial radars depends on the method of observing the airspace used in the radar.

The purpose of the article is to analyse the possibilities for increasing the quality of the radar information system and to increase the accuracy of the 2D coordinate estimation as a result of determining the airspace in a low-base MIMO radar based on two-coordinate radar.

\section{MAIN MATERIAL}

In this paper, we will consider the MIMO-radar system, which is built on the basis of monostatic two-coordinate active observation radar, which are united through a data exchange network and have a single coordinate scale and synchronized in time. The two-coordinate radar measures two polar coordinates of the airspace (azimuth and sloping range). Radar information from radars is transmitted to a compatible data processing station.

Now radars are combined into systems with distributed data processing. In the central processor of the network from radars arriving aircraft tracks. On the received tracks of planes in the central processor the uniform route for each aircraft is formed. The disadvantages of the distributed system are that the time of detecting the route of the aircraft is determined by the time it is detected in separate radar. The advantage of such a system is the low requirements for data transmission lines, since there is no false radar information and only the true routes of the aircraft are transmitted.

In a system with centralized data processing in the central process of the network from radars receive marks of planes, and not trajectories. After processing the received marks, a single trajectory for each aircraft is formed. The disadvantage of such a system is the high requirements for data transmission lines, since a large number of false marks are transmitted to the central processor along with the actual aircraft markings. The advantage of such a system is to reduce the time to detect the route of the aircraft and increase the accuracy of its support by increasing the pace of data updating.

Features of the construction of separate radar equipment determine the methods of processing radar information, which can be implemented in the MIMO system. The quality of radar information is determined by the multiplicity of overlap areas.

For example, analyse for a spatially coherent MIMO radar system consisting of radar of the same type, spaced at a distance $\mathrm{L}$. The overlap area is formed when synchronous rotation of radars with diagrams of antenna beams due to location on a small base (Figure 1).

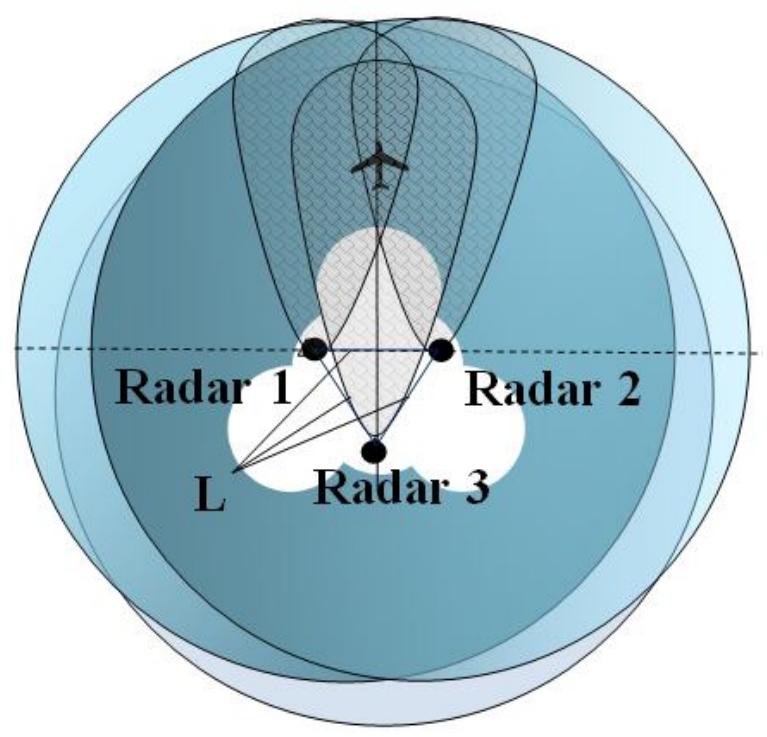

Figure 1: The area, where there is overlapping of the beams pattern of radars with synchronous rotation in the MIMO-radar when the base equal $3 \mathrm{~km}$

Possibilities of introducing multi-site radar methods to detect and determine the location of airborne objects in the MIMO-radar system depend on the simultaneous observation of the airborne object by all radars. There are no flexible or multichannel scans in the system. Radar only uses the mechanical rotation of the antenna, so the implementation of a coordinated space review will be provided by synchronous viewing of space. 
In this case, the functioning of the MIMO-radar system in the mode of autonomous co-operative reception is possible using the method of measuring the range of determining the coordinates of air objects. In this case, there are a number of additional capabilities associated with the use of radar data processing techniques in multi-site radar systems [11]-[12]:

- definition of the third coordinate of the patch for the MIMO radar system, consisting of at least three two-dimensional radar;

- increase the probability of detecting an aircrafts in a certain period of time compared to autonomous radar;
- increase the accuracy of determining the coordinates of an air object by means of averaging or aggregation of data in the MIMO-radar.

The use of small base systems will simplify the technical implementation of the MIMO radar system[11]-[12]. Such a system will provide spatial coherence with the ability to combine the processing of radar information. There is no need to create a fundamentally new system, only combine existing radars. It is cost effective and relatively fast.

On Figure 2 the system design is presented. In this case, it is possible using the method of measuring the range for determining the coordinates [11]-[12].

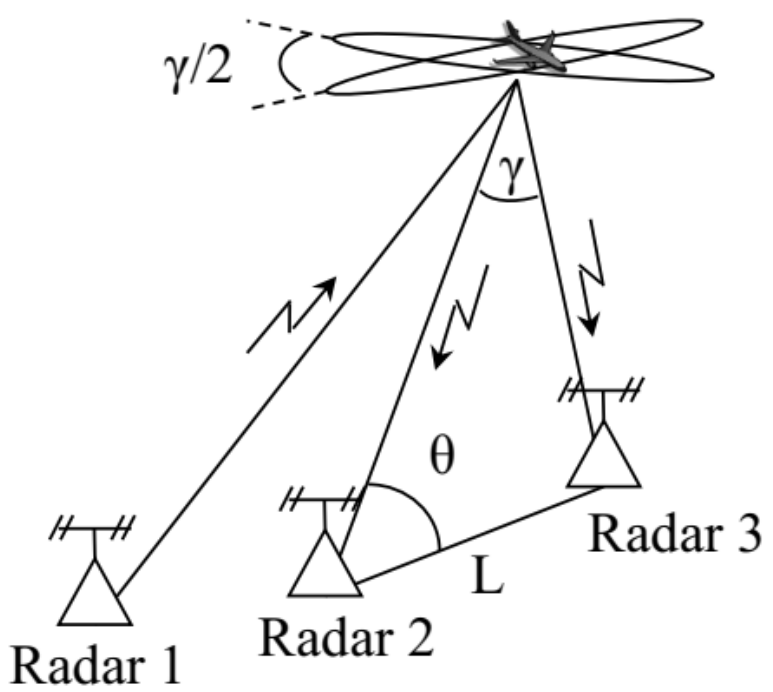

b)

Figure 2: The improving accuracy of aircrafts coordinates by the MIMO-radars in such case: a) the autonomous receiving MIMO-radar; b) the MIMO-radar with cooperative receiving

Provided that the radar locations are known with high accuracy, the primary parameters of the airborne object, which are measured by the radar and used for the local determination of the air object, are the range of the air object to the corresponding radar (R1, R2, R3).

An important advantage of applying MIMO-radar for determining the coordinates of aircraft is the ability to provide the required accuracy of the determination of coordinates of aircraft without the use of accurate measurement of angular coordinates in radar. This reduces the requirements for the antenna beam chart width and size. Reducing the size of the antennas allows to reduce the cost of the radar.

In MIMO-radars, due to system effects, it is possible to negate the errors by angular coordinates by measuring only distances (distances), without using angular measurements, explains Figure 2.

Ellipse errors are substantially flattened in range. This is due to the fact that the accuracy of determining the azimuth aircrafts in the radar is much worse than the accuracy of the definition of range of aircrafts. When the data is processed from two radar systems, the ellipse error corresponds to the crossing of the each radar ellipses error. Due to this, the accuracy of determining the coordinates of aircrafts can be greatly improved. This is equivalent to improving the accuracy of azimuth measurement by measuring the range.

When implementing the range method for determining the coordinates of aircrafts in the two-position MIMO-radar (Figure 2,a), the mean square error of determining the azimuth aircrafts is estimated by the expression (1):

$$
\sigma_{\theta}=\frac{\sigma_{\mathrm{R}} \sqrt{2}}{\mathrm{~L} \sin (\theta)},
$$

where $\sigma_{\theta}-$ mean square error of azimuth determination of aircraft; 
Hennadii Khudov et al., International Journal of Emerging Trends in Engineering Research, 8(5), May 2020, 2026 - 2030

$\mathrm{L}$ - the base between radars;

$\sigma_{\mathrm{R}}$ - the mean square error of range determination of the aircraft;

$\theta$ - the azimuth an aircraft relative to radar.

When implementations of the range finding method for determining the coordinates of aircrafts (Figure 2,b), the estimation of the mean square error for determining the azimuth of aircrafts by measuring the range is calculated by the expression (2):

$$
\sigma_{\theta}=\frac{\sigma_{\Sigma_{R}} \sqrt{2}}{L \sin (\theta)}=\frac{2 \sqrt{2} \sigma_{R}}{L \sin (\theta)},
$$

where $\sigma_{\Sigma_{\mathrm{R}}}-$ mean square error of the range finding determination of aircraft.

From the comparison of expressions (1) and (2), it follows that the mean square error determination of azimuth using the range finding method for determining the coordinate of aircrafts is twice as large as when using the range method of determining the coordinates of aircrafts. This is equivalent to reducing the size of the database twice.

In the general case, if in the MIMO-radar the number of primary measurements (distances, total distances) exceeds the minimum required amount needed to determine the coordinates of the aircraft, then excessive measurements can be used to improve the accuracy of the determination of the coordinates of the aircraft.

\section{CONCLUSION}

The conclusions from the analysis are as follows: the using of multistatic methods in MIMO-radar to improve the accuracy of determining the $2 \mathrm{D}$ coordinates of the aircrafts is possible. The possibility of improving determining the $2 \mathrm{D}$ coordinates provided at the intersection of the detection zones of the MIMO-radar system, which consists of two-dimensional observations radar. The informativity of such a MIMO-radar system will be increased. However, the accuracy of determining the coordinates in measuring the range depends on the mean square error of measuring the range of individual radars, the size of the base, the position of the air object in the zone from the base, the distance air objects from the autonomous radar system.

\section{REFERENCES}

1. A. Farina, A. De Maio, and S. Haykin, The Impact of Cognition on Radar Technology, Stevenage: SciTech Publishing Inc., 2017,

2. P. Shchypanskyi, V. Savchenko, O. Martyniuk, and I. Kostiuk, Air Defense Planning from an Impact of a Group of Unmanned Aerial Vehicles based on Multi-Agent Modeling, IJETER, Vol. 8., № 4, 2020, pp. 7-12.

DOI: https://doi.org/10.30534/ijeter/2020/59842020.
3. I. Ruban, H. Khudov, V. Lishchenko, A. Zvonko, S. Glukhov, I. Khizhnyak, V. Maliuha, Y. Polonskyi, R. Kushpeta, The Calculating Effectiveness Increasing of Detecting Air Objects by Combining Surveillance Radars into The Coherent System, IJETER, Vol. 8., № 4, 2020, pp. 1295-1301.

DOI: https://doi.org/10.30534/ijeter/2020/58842020.

4. V. Vasylyshyn Adaptive variant of the surrogate data technology for enhancing the effectiveness of signal spectral analysis using eigenstructure methods, Radioelectronics and Communications Systems, 2015, Vol. 58, pp. 116-126. DOI: https://doi.org/10.3103/S0735272715030036.

5. H. Khudov, S. Kovalevskyi, A. Irkha, V. Lishchenko, O. Serdiuk and F. Zots, The Proposals for Synchronization Positions of MIMO Radar System on the Basis of Surveillance Radars, in Intern. Scient.-Pract. Conf. Problems of Infocommunications. Science and Technology (PIC S\&T), 2019, pp. 547-551. DOI: https://doi.org/10.1109/PICST47496.2019.9061284.

6. H. Khudov, I. Khizhnyak, V. Koval, V. Maliuha, A. Zvonko, V. Yunda, V. Nagachevskyi, and V. Berezanskyi The Efficiency Estimation Method of Joint Search and Detection of Objects for Surveillance Technical Systems, IJETER, Vol. 8. № 3, 2020, pp. 813-819.

DOI: https://doi.org/10.30534/ijeter/2020/34832020

7. H. Khudov, A. Zvonko, S. Kovalevskyi, V. Lishchenko, and F. Zots. Method for the detection of smallsized air objects by observational radars, EEJET, № 2/9 (92), 2018, pp. 61-68.

DOI: https://doi.org/10.15587/1729-4061.2018.126509.

8. H. Khudov, A. Zvonko, I. Khizhnyak, V. Shulezko, V. Khlopiachyi, V. Chepurnyi, and I. Yuzova. The Synthesis of the Optimal Decision Rule for Detecting an Object in a Joint Search and Detection of Objects by the Criterion of Maximum Likelihood, IJETER, № 8(2), 2020, pp. 520-524.

DOI: https://doi.org/10.30534/ijeter/2020/40822020.

9. V. Lishchenko, H. Khudov, V. Tiutiunnyk, V._Kuprii, F._Zots, and G._Misiyuk. The Method of Increasing the Detection Range of Unmanned Aerial Vehicles In Multiradar Systems Based on Surveillance Radars, in 2019 IEEE 39th International Conference on Electronics and Nanotechnology (ELNANO), 2019. DOI: https://doi.org/10.1109/ELNANO.2019.8783263.

10. H. Khudov, I. Khizhnyak, I. Yuzova, O. Baranik, G. Semiv, S. Bondarenko, and O. Tytarenko. The Optimization Technique for Joint Discrete Search and Detection of Observation Objects, International Journal of Emerging Trends in Engineering Research, № 8(2), 2020, pp. 533-538. DOI: https://doi.org/10.30534/ijeter/2020/42822020.

11. H. Khudov, A. Fedorov, D. Holovniak, and G. Misiyuk. Improving the Efficiency of Radar 
Hennadii Khudov et al., International Journal of Emerging Trends in Engineering Research, 8(5), May 2020, 2026 - 2030

Control of Airspace with the Multilateration System Use, in Intern. Scient.-Pract. Conf. Problems of Infocommunications. Science and Technology (PIC S\&T), 2018, pp. 680-684. DOI: https://doi.org/10.1109/infocommst.2018.8632 141.

12. V. Lishchenko, V. Chaliy, H. Khudov, and A. Zvonko. Proposals for Improving of Air Surveillance Informativity in MIMO Radar Systems Based on Two-Dimensional Radars, in

Intern. Scient.-Pract. Conf. Problems of Infocommunications. Science and Technology (PIC S\&T), 2018, pp. 153-156. DOI: https://doi.org/10.1109/infocommst.2018.8632052. 